\title{
Joint space width measures cartilage thickness in osteoarthritis of the knee: high resolution plain film and double contrast macroradiographic investigation
}

\author{
J Christopher Buckland-Wright, Diana G Macfarlane, John A Lynch, M Kris Jasani, \\ Charles R Bradshaw
}

\begin{abstract}
Objective-To test reliability of joint space width (JSW) measurements as a predictor of cartilage thickness in knees of patients with osteoarthritis (OA), using high definition microfocal radiography.

Method-JSW was measured from weight bearing plain film macroradiographs taken in the tunnel view and compared with the sum of femoral and tibial cartilage thicknesses measured from double contrast macroarthrograms of the same regions of the same knees obtained in the non-weight bearing lateral position.

Results-All knees had medial compartment OA. Comparison of the JSW with the sum of the tibial and femoral cartilage thicknesses revealed a highly significant correlation $(p<0.0001)$ between the two measurements in the medial but not the lateral compartment. In the middle region of both compartments, JSW was smaller than the cartilage thickness, indicating that, on standing, the curvature of the femoral condyles compressed the cartilage in this region.
\end{abstract}

Conclusions-JSW reliably measured cartilage thickness in the medial but not the lateral compartment of knees with medial compartment OA. Depending upon the stage of OA disease, JSW reliably reflects cartilage thinning and compression.

(Ann Rheum Dis 1995; 54: 263-268)

Joint space width (JSW) measurement is used as a major criterion in the diagnosis of osteoarthritis (OA) from radiographs and for monitoring progression of the disease. ${ }^{1}$ Doubt has been cast on the reliability of this approach by studies in which the radiographic status of the joint was compared with the arthroscopic appearance of the cartilage surface. ${ }^{23}$ The investigators found that joint space often appeared normal in patients who had severe cartilage loss, or narrowed in knees with normal cartilage. ${ }^{2} 3$ Recently, we have formally evaluated the usefulness of the standing semiflexed and weight bearing or modified tunnel view for the detection of joint space narrowing in patients with knee OA. ${ }^{4}$ Three patterns of joint space narrowing were evaluated, as observed in both views $(30 \%)$, in only the standing semiflexed view (8\%), and in only the weight bearing tunnel view $(22 \%)$.

The aim of this study was to verify if JSW in the weight bearing tunnel view reliably measures cartilage thickness in OA. Instead of using arthroscopy, which mainly visualises the surface appearance of cartilage, we undertook a double contrast radiographic study to visualise and measure the thickness of the tissue from high definition macroradiographs. ${ }^{5}$ Compared with conventional radiography, which has been shown to have a fairly large coefficient of variation for JSW measurement, ${ }^{78}$ macroradiography provides accurate and reproducible measurements of $x$ ray features based on standardised radiographic and mensural procedures. ${ }^{568}$

JSW was measured from plain film macroradiographs taken in the weight bearing or modified tunnel view. It was compared with the sum of the tibial and femoral cartilage thicknesses measured from double contrast macroarthrograms of the same knee radiographed at the same angle but in the nonweight bearing lateral position. Initially, macroradiographs were obtained in the weight bearing tunnel position for such comparisons, but the procedure was abandoned as it resulted in the appearance of a fluid level in the joint, rendering it impossible to recognise the cartilage boundaries for purposes of thickness measurements.

\section{Patients and methods}

PATIENTS

We studied 20 patients (six male) with a mean age of 58.1 (range 35-74) years, a mean disease duration (based on the pain in the worst, most painful knee) of 5.7 (range 3-20) years, and a mean weight of 73.4 (range 54-104) kg. Patient selection was based upon clinical and radiographic criteria. The status of the study knee was graded using the Kellgren and Lawrence criteria. ${ }^{9}$ Exclusion criteria included evidence of other types of arthritis, previous trauma, surgical intervention, or treatment with corticosteroids. All patients were seronegative for rheumatoid factor and had an erythrocyte sedimentation rate within the normal range. 
As ethical considerations precluded subjecting non-diseased age and sex matched hospital attendees to radiography, macroradiographs of 14 healthy, non-arthritic volunteers (seven men and seven women, mean age 35.5 (range 23-56) years; mean weight $73 \cdot 2$ (range 60-89) $\mathrm{kg}$ ) were obtained from medical and laboratory staff. Radiographically, the knees of all reference subjects were devoid of both osteophytes and sclerosis. The dimensions of their joint space width provided a reference range for the distance between bones in anatomically normal healthy knee joints of subjects with a body weight similar to that of the patients. These JSW data are referred to as the reference values and were used to define the degree to which JSW was narrowed in the OA knees. The difference in the mean age, of approximately 20 years, between the reference subjects and OA patients might result in some joint space loss in the latter, due to age. Whether age related changes are significant between the groups in this study, remains speculative since significant changes have been reported only in studies in which the ages of the subjects were between 10 and 86 years and between 22 and 78 years. ${ }^{11}$

PLAIN FILM MACRORADIOGRAPHY IN THE WEIGHT BEARING TUNNEL VIEW

Stereopair macroradiographs at $\times 5$ magnification $^{56}$ were taken of the most painful kneethat is, the one selected for the arthrographic investigation. The knee was radiographed at $130^{\circ}$ angle in the weight bearing or modified tunnel view, ${ }^{412}$ which involved the patient sitting on the edge of a stool with weight transmitted through the leg undergoing $x$ ray examination. The joint was radiographed in the anteroposterior position with the patient's foot placed in a slot on the patient table. ${ }^{412}$ The $130^{\circ}$ angle of flexion used in this study is the same as that used for obtaining the conventional non-weight bearing tunnel view. ${ }^{13}{ }^{14}$ This angle was checked by means of a Perspex template. With the aid of the laser and image intensifier screening, the position of

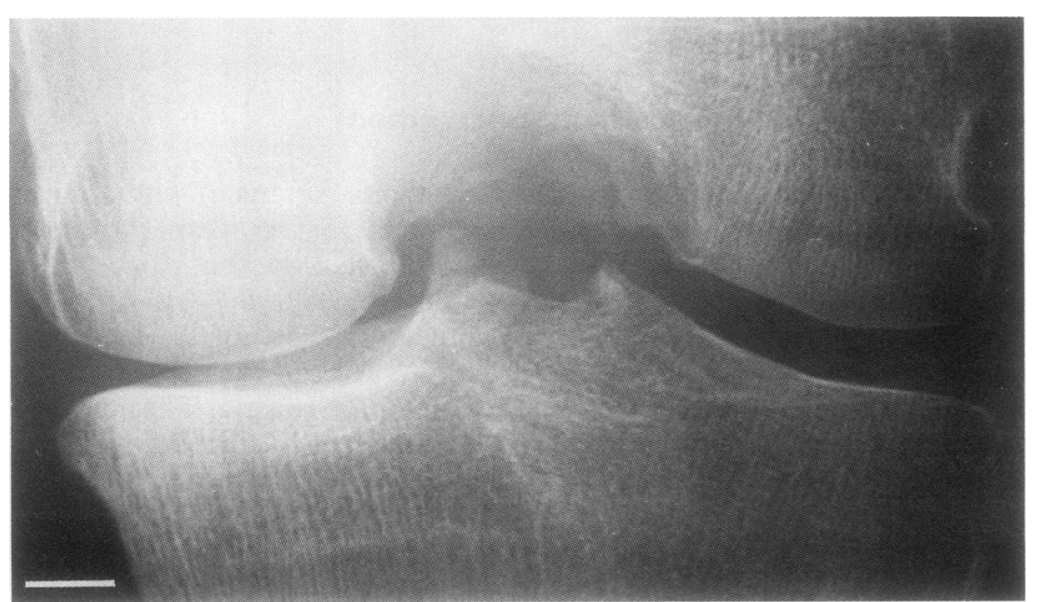

Figure 1 Plain film macroradiograph of an osteoarthritic knee in the load bearing tunnel view showing the position in which the joint was radiographed at $\times 5$ magnification. Horizontal bar represents $41 \mathrm{~mm}$. the knee was adjusted to ensure that the tibial plateau was horizontal and perpendicular to the radiographic plate and that the tibial spines were centrally placed relative to the femoral notch (fig 1). The view assessed alteration to cartilage thickness over the popliteal surface of the condyles.

DOUBLE CONTRAST MACROARTHROGRAPHY IN THE NON-WEIGHT BEARING LATERAL POSITION Niopam 200 (E Merck Ltd, Hampshire, UK) was chosen as the contrast medium for the arthrographic examination, as an earlier study $^{15}$ had shown that a contrast medium containing iodine $200 \mathrm{mg} / \mathrm{ml}$ provided better definition of the articular cartilage in macroradiographs of the knee than contrast media with the greater iodine concentrations used conventionally.

After injection of a local anaesthetic, fluid found in the joint was aspirated. Five to $10 \mathrm{ml}$ of the contrast medium was injected into the joint cavity, followed by $40-80 \mathrm{ml}$ of air. A bandage was then wrapped tightly around the leg immediately above the patella in order to restrict both medium and air from entering the suprapatellar pouch. After injection of the contrast medium and air, the knee was flexed and extended several times to spread the medium over the inner surface of the joint. This process was further aided by the patient walking from the examination bed to the $x$ ray unit.

For radiography, the patient lay on the table in the lateral position with the joint space to be examined uppermost. By flexing the knee to $130^{\circ}$ the joint space was spread open to display the intra-articular components. With the knee resting in this position, anteroposterior stereopair macroradiographs (magnification $\times 7$ to $\times 9$ ) of the medial and lateral tibiofemoral compartments were obtained at the same angle as in the weight bearing modified tunnel view. As already explained, it was necessary to obtain the macroarthrograms in the non-weight bearing position to avoid the appearance of a fluid level in the joint. The remaining details were as already described for preparation of the stereopair macroradiographs in the tunnel view of the joint.

\section{METHODS OF ASSESSMENT}

Qualitative assessment. The stereopair macroradiographs obtained were examined, by a single observer, under a Large Format Stereoscope (Ross Instruments, Salisbury, UK), which permitted a three dimensional evaluation of the joint structure. ${ }^{56}$ Articular cartilage damage was graded as none (smooth cartilaginous surface with no apparent thinning), mild (minimal thinning and irregularity), moderate (large localised defects and moderate thinning and irregularity), or severe (severe denudation of cartilage). Its extent was judged by assessing whether it was confined to only the outer, middle or inner thirds of the femoral and tibial cartilages in the medial and lateral compartments. Additional 


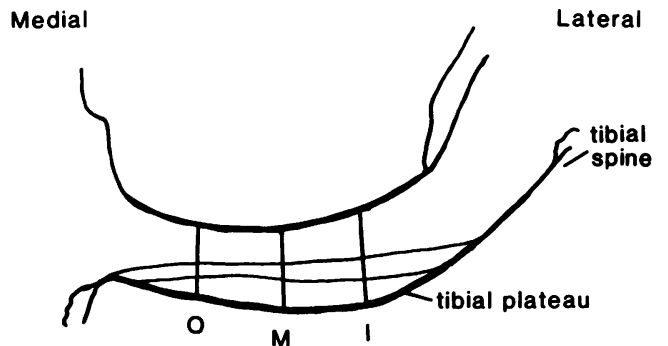

A

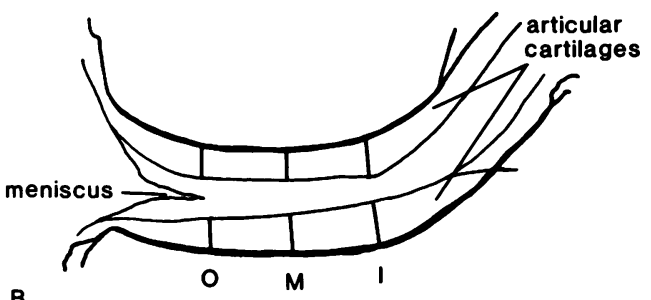

Figure 2 Diagrams of the medial tibiofemoral compartment of an osteoarthritic knee illustrating the three sites at which macroradiographic measurements were carried out for determining the joint space width in the tunnel view of the knee joint $(A)$ and the femoral and tibial articular cartilage thickness from the double contrast macroarthrogram (B). Measurements were taken across the outer (O), middle (M) and inner (I) one third of both the medial and lateral compartments at the joint.

observations included the extent of osteophytosis, ${ }^{4}$ subchondral sclerosis ${ }^{4}$ and meniscal cartilage damage. Using a four point scale, the meniscal cartilages were graded as not damaged (normal contour without evidence of swelling or tear), midly damaged (localised meniscal swelling or tear), moderately damaged (moderate size tear or degeneration), or severely damaged (extensive tear or disintegration).

Quantitative assessment. For this procedure, the same observer examined the stereopair macroradiographs under the stereoscope. The right hand, back illuminated carriage comprised a digitiser tablet linked to an MOPVideoplan (Zeiss, Hertfordshire, UK). ${ }^{16}$

A detailed description of the method of measuring the $x$ ray features and its accuracy in recording them is reported elsewhere; ${ }^{16}$ its coefficient of variation for linear JSW measurements is $3 \cdot 7 \% .^{16}$ The data, which were

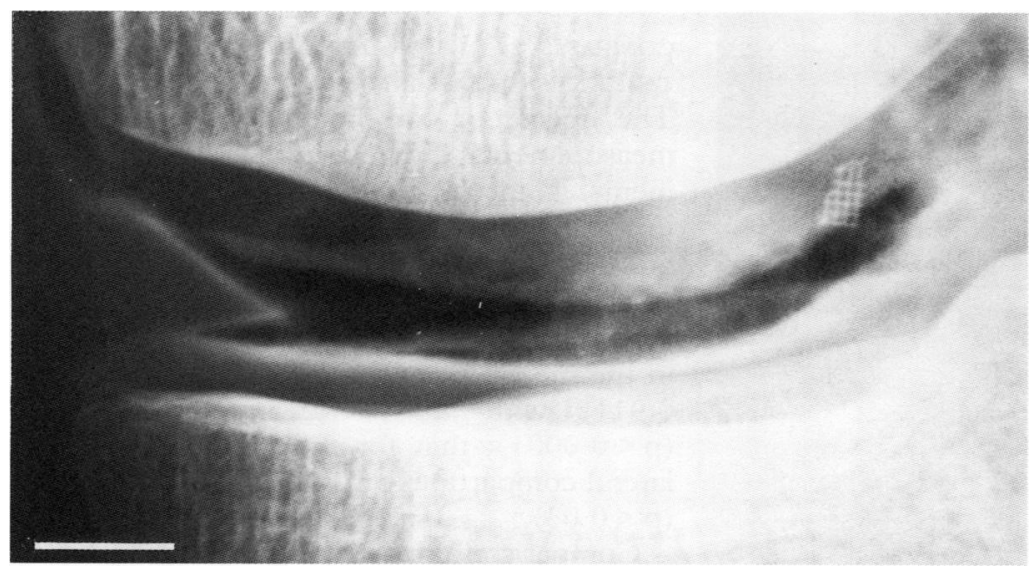

Figure 3 Part of the double contrast macroarthrogram of the medial compartment of an osteoarthritic knee taken at $\times 8$ magnification, showing contrast medium imbibition in the tibial and meniscal cartilages-changes that we have found to be associated with cartilage fibrillation and fissuring. ${ }^{15}$ The wire grids visible in this and the other macroarthrograms were used to determine the radiographic magnification. Hoizontal bar represents $36 \mathrm{~mm}$. initially recorded on microcomputer disks, were transferred to an IBM PC/AT computer and combined into a single large data file. Statistical analysis was carried out using the SPSS/PC+ package. ${ }^{17}$

\section{MEASUREMENTS}

foint space width was measured using the macroradiographs of the weight bearing tunnel view of the knee. As shown in figure 2 , JSW (defined as the interbone distance) was measured (in millimetres) at three sites along the joint margin of both the medial and lateral tibiofemoral compartments. The sites were obtained by subdividing the articulating surface of the joint into four and the measurements were taken at the quarter, mid and three-quarter divisions.

Articular cartilage thickness was measured using the double contrast macroarthrograms. It was represented by the distance between the articular surface, identified by the presence of a thin layer of contrast medium over and within it (fig 3), and the mineralised osteochondral junction. This distance was measured (in millimetres) at the same sites over the femoral condyles and tibial plateaux as those chosen for the measurement of JSW in the plain film macroradiographs and shown in figure 2.

ANALYSIS OF THE DATA

Comparability of JSW and articular cartilage thickness measurements made at the individual sites in the two compartments was assessed using the Wilcoxon matched pairs test. Comparison of each parameter between the reference and OA knees was assessed using the Mann-Whitney test. The degree of correlation between JSW and articular cartilage thickness within the medial and lateral knee compartments of the OA patients was assessed using Pearson's correlation coefficient $(r)$ and the non-parametric Kendall's Tc test methods. The latter test was used because of its greater power in determining the degree of association between parameters which may not have a strict linearity of fit. ${ }^{18}$ For all tests, $p$ values less than 0.05 were considered statistically significant.

\section{Results}

Qualitative assessment of the plain films revealed 11 knees were Kellgren and Lawrence grade I, six were grade II, and three grade III; there were none at grade IV.

PLAIN FILM MACRORADIOGRAPHY IN THE WEIGHT BEARING TUNNEL VIEW

Osteophytes that were doubtful on conventional radiography were clearly visible on macroradiography. The latter revealed that osteophytes and subchondral sclerosis were present in the medial compartment of all 20 OA knees. In the lateral compartment osteophytes were present in seven and subchondral sclerosis in 15. 
Table 1 foint space width ( $(S S W)$ measured from weight bearing tunnel view macroradiographs of healthy knees from the reference group of non-arthritic volunteers and patients with knee $O A$, together with the sum of femoral and tibial cartilage thicknesses measured from the double contrast macroarthrograms of the $O A$ knees without weight bearing

\begin{tabular}{|c|c|c|c|}
\hline & \multicolumn{2}{|l|}{ Loaded $7 S W(\mathrm{~mm})$} & \multirow{2}{*}{$\begin{array}{l}\text { Sum of femoral and tibial } \\
\text { cartilage thicknesses (mm) } \\
\text { OA knees } \\
(n=20)\end{array}$} \\
\hline & $\begin{array}{l}\text { Healthy knees } \\
(n=14)\end{array}$ & $\begin{array}{l}\text { OA knees } \\
(n=20)\end{array}$ & \\
\hline \multicolumn{4}{|c|}{ Medial compartment } \\
\hline Outer & $4 \cdot 45(4 \cdot 14$ to $4 \cdot 76)$ & $3.21(2.54 \text { to } 3.88)^{\star}$ & $3.00(2.35$ to 3.65$)$ \\
\hline Middle & $4.55(4.15$ to 4.95$)$ & $3.35(2.65 \text { to } 4.05)^{\star \star}$ & $3.70(2.96$ to 4.44$)$ \\
\hline Inner & $5.77(5.33$ to 6.20$)$ & $4.56(4.00 \text { to } 5 \cdot 12)^{\star \star \star}$ & $4 \cdot 75(4 \cdot 11$ to $5 \cdot 39)$ \\
\hline \multicolumn{4}{|c|}{ Lateral compartment } \\
\hline Outer & $5.46(4.88$ to $6 \cdot 04)$ & $4.74(4.03$ to 5.45$)$ & $4.50(3.70$ to 5.30$)$ \\
\hline Middle & $5.20(4.68$ to $5 \cdot 72)$ & $5 \cdot 15(4.51$ to $5 \cdot 79)$ & $6 \cdot 10(5.60$ to 6.60$)$ \\
\hline Inner & $5.66(5.09$ to 6.22$)$ & $5.04(4.55$ to 5.53$)$ & $4.61(3.89$ to 5.33$)$ \\
\hline
\end{tabular}

Values are mean $(95 \%$ confidence interval). Significant differences between healthy and $\mathrm{OA}$ knees: ${ }^{\star} \mathrm{p}<0.0008 ;{ }^{\star \star} \mathrm{p}<0.01{ }^{\star \star \star} \mathrm{p}<0.006$ (Mann-Whitney test)

Foint space width measurements. Compared with the reference range, the mean JSW in OA knees was significantly decreased in the medial, but not in the lateral compartment (table 1). It was decreased at all the three measurement sites: $p<0.0008$ for the outer, $p<0.01$ for the middle, and $\mathrm{p}<0.0006$ for the inner site of the joint margin (Mann-Whitney test) (table 1).

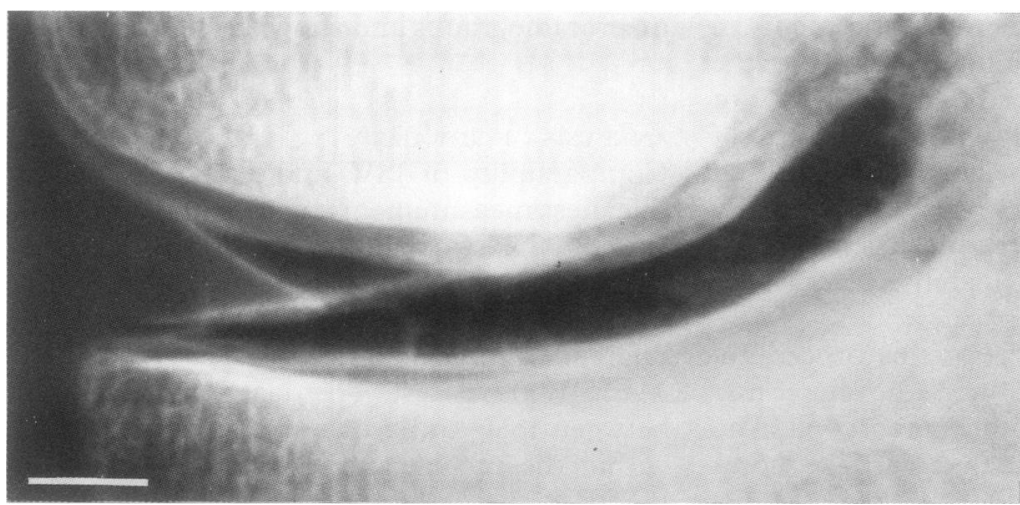

Figure 4 Part of a double contrast macroarthrogram of the medial compartment of an osteoarthritic knee taken at $\times 7$ magnification, showing pronounced cartilage thinning over the outer region of the tibial plateau and less severe thinning in the same region on the femoral condyle. Horizontal bar represents $28 \mathrm{~mm}$.

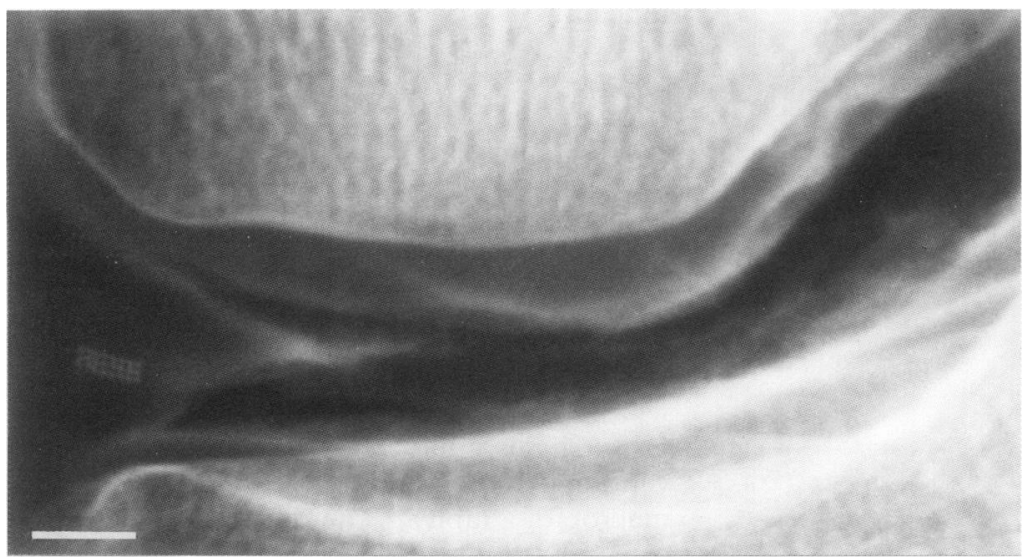

Figure 5 Part of the double contrast macroarthrogram of the medial compartment of an osteoarthritic knee taken at $\times 6$ magnification, showing cartilage thinning in the outer third of the tibial plateau and femoral condyle. There is marked imbibition of contrast medium in both articular cartilages, several focal lesions on the femoral cartilage, and damage to the meniscus. Horizontal bar represents $18 \mathrm{~mm}$.
Table 2 Double contrast macroradiographic findings of the number of osteoarthritic knees with meniscal and articular cartilage damage

\begin{tabular}{|c|c|c|c|c|}
\hline & \multicolumn{4}{|c|}{ Grade } \\
\hline & None & Mild & Moderate & Severe \\
\hline \multicolumn{5}{|c|}{$\begin{array}{l}\text { Articular cartilage damage } \\
\text { Medial compartment }\end{array}$} \\
\hline Femoral cartilage & - & 13 & 3 & 4 \\
\hline Tibial cartilage & - & 10 & 4 & 6 \\
\hline \multicolumn{5}{|l|}{ Lateral compartment } \\
\hline Femoral cartilage & - & 16 & 3 & 1 \\
\hline Tibial cartilage & - & 14 & 6 & - \\
\hline \multicolumn{5}{|l|}{ Meniscal damage } \\
\hline Medial meniscus & 6 & 5 & 4 & 5 \\
\hline Lateral meniscus & 9 & 7 & 3 & 1 \\
\hline
\end{tabular}

* See text for explanation of grading scales.

DOUBLE CONTRAST MACROARTHROGRAPHY IN THE NON-WEIGHT BEARING LATERAL POSITION

Meniscal and articular cartilage damage. Table 2 summarises the qualitative results of the meniscal and articular cartilage damage. Both articular cartilage and menisci were more severely damaged in the medial than the lateral compartments. In the medial compartment, damage to the meniscus was similar to that on the surface of the tibial cartilage.

Imbibition of contrast medium, observed as a bright radiodense band on the surface of articular cartilage, was more extensive in cartilage with moderate to severe (figs 4-6) compared with mild damage (fig 3 ). In joints where cartilage was grossly thinned and absent (fig 7), contrast medium had entered the subchondral region.

Articular cartilage thickness measurements. Across the medial compartment of OA knees there was a gradient of articular cartilage thinning (table 1 , figs 4-7): on average it was thinnest at the outer site, intermediate at the middle site, and thickest at the inner site on both tibial and femoral surfaces (table 1). The differences between sites were more pronounced on the tibial than the femoral surfaces. By contrast, in the lateral compartment (table 1) the cartilage was on average thickest at the middle site on both the joint surfaces.

COMPARISON OF JSW AND CARTILAGE

THICKNESS MEASUREMENTS IN OA KNEES

The degree of correlation between the two measurements differed in the two compartments. They were strongly correlated at all three sites in the medial compartment (fig 8A), but only at the middle site in the lateral compartment, where the correlation was weaker (fig 8B). The Pearson $r$ values for the three sites in the medial compartment were greater than 0.911 , with a Tc greater than 0.607 $(\mathrm{p}<0.0001)$; that for the middle site in the lateral compartment was 0.657 , with Tc 0.305 $(\mathrm{p}<0.03)$.

Comparison of the two parameters at individual sites within the two compartments showed the JSW to be significantly smaller than the sum of the femoral and tibial cartilage thicknesses over the middle, but not over either 


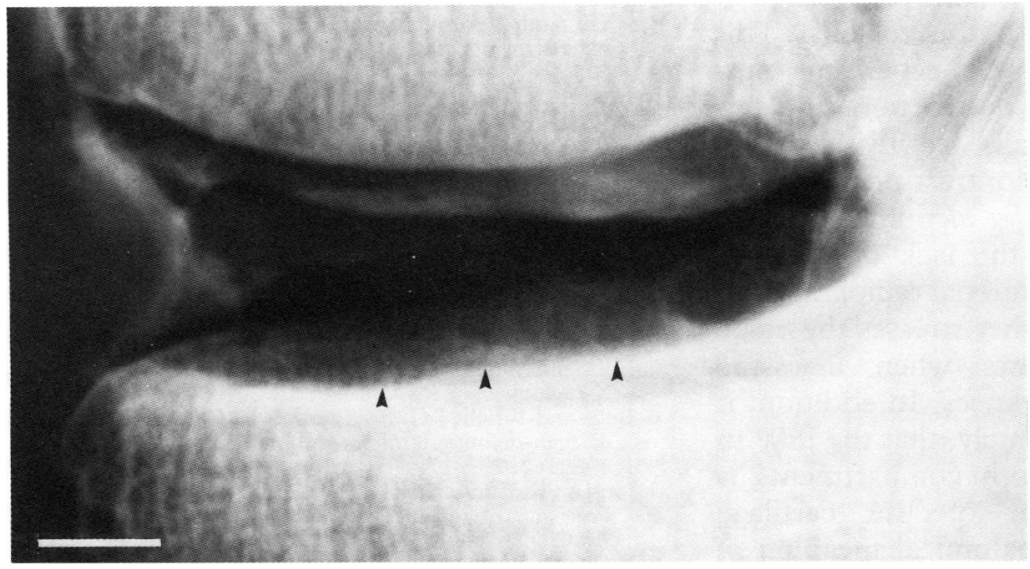

Figure 6 Part of a double contrast macroarthrogram of the medial compartment of an osteoarthritic knee taken at $\times 7$ magnification, showing total articular cartilage loss from the outer third and pronounced thinning over the middle region of the tibial plateau (arrowed). On the femoral condyle the surface of the cartilage is irregular and shows thinning more clearly over the outer region. The free edge of the meniscus is badly damaged. Osteophytes are present at the condyle and tibial margins. Horizontal bar represents $30 \mathrm{~mm}$.

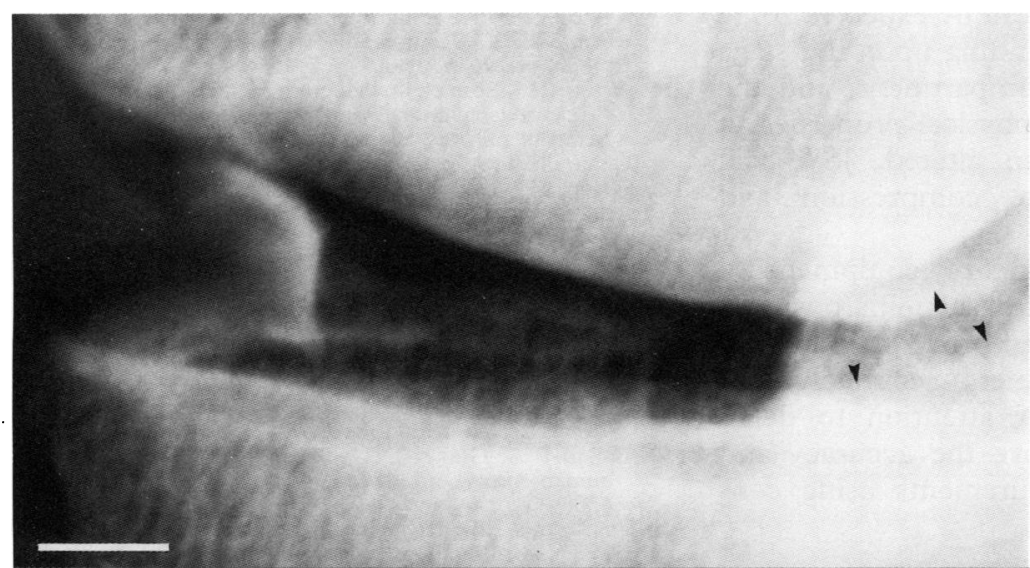

Figure 7 Part of a double contrast macroarthrogram of the medial compartment of an osteoarthritic knee taken at $\times 7.5$ magnification, showing exposed subchondral bone over the outer and middle regions of the femoral tibial surfaces. A remnant of articular cartilage is visible at the inner region of the tibia and at the inferior margin of the femoral notch (arrowed). The femoral condyle is overlayed by a soft tissue layer rich in contrast medium. Breaks in the femoral cortex are present, permitting contrast to enter the subchondral bone. The meniscus has been severely damaged and truncated. Horizontal bar represents $32 \mathrm{~mm}$. of the other sites $(p<0.001$ and $<0.002$ for the medial and the lateral compartments, respectively).

\section{Discussion}

Comparison of the JSW obtained from weight bearing tunnel view macroradiographs of OA knees with the sum of the tibial and femoral cartilage thicknesses measured from macroarthrograms of the same knees in the nonweight bearing lateral position revealed a highly significant correlation between the two measurements in the medial but not the lateral compartment. Thus JSW reliably measures cartilage thickness in the medial compartment of OA knees.

The present study indicates that, in OA, cartilage thinning occurs in a gradient across the joint surface: the thinning was most pronounced, in all patients, over the outer and least over the inner regions of the joint surface. Thinning at the outer region was greater on the tibial plateau than on the femoral condyle-an observation that is consistent with there being a greater load per unit area at the concave tibial compared with the convex femoral articular surfaces, ${ }^{19-21}$ and the with the occurrence of meniscal damage in virtually all the patients. Under these circumstances the menisci can no longer be assumed to have shock absorbing functions. ${ }^{22}$

In the lateral compartment of the knee, although there was a poor correlation between JSW and the sum of the cartilage thicknesses across the compartment as a whole (fig $8 \mathrm{~B}$ ), this was not the case in the middle region. Comparison with the reference value for healthy knees showed that there was little if any thinning. However, cartilage thinning was present in the outer and inner thirds, but only in the group of patients who already had advanced joint space narrowing in the medial compartment. The arthrogram confirmed that in the knees studied cartilage damage was present over either the outer or the inner thirds of the femoral and tibial surfaces, but not
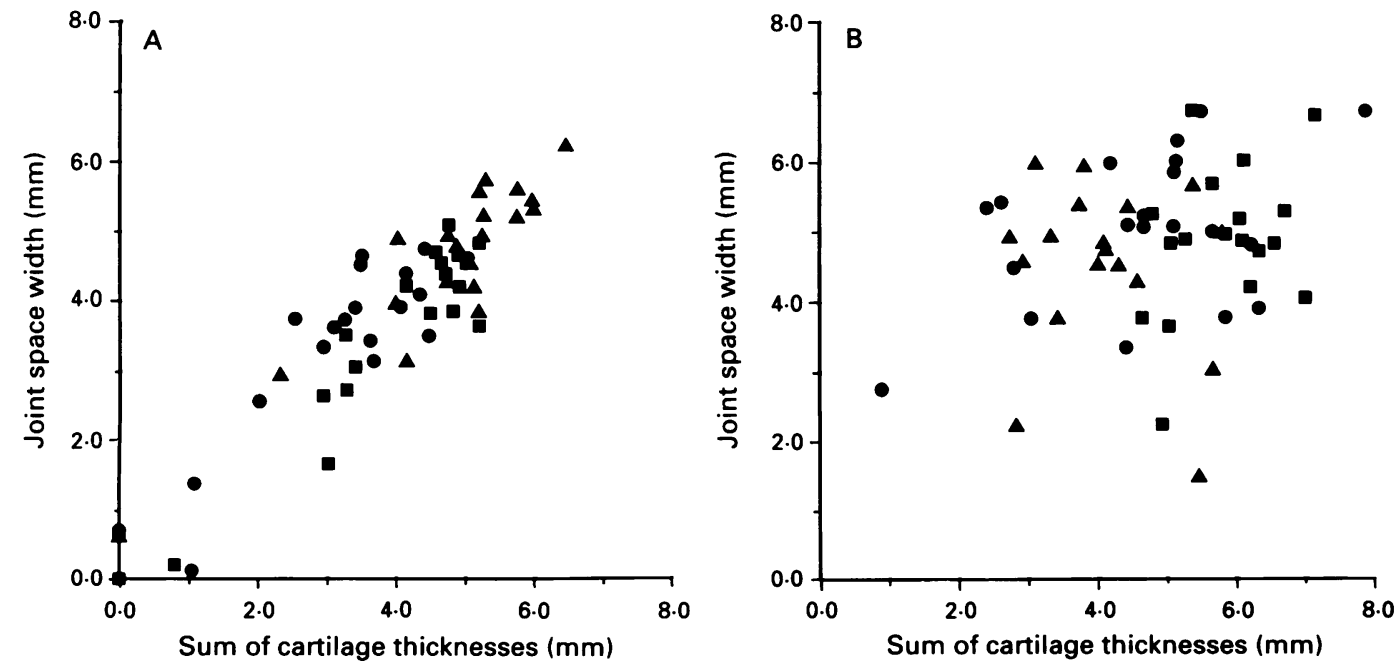

Figure 8 Comparison of the joint space width from plain film macroradiographs and the sum of femoral and tibial articular cartilage thicknesses measured from the double contrast macroradiographs at the chosen outer ( $\bullet)$, middle (ט) and inner $(\mathbf{\Delta})$ sites of the joint in $(A)$ the medial and $(B)$ the lateral tibiofemoral compartments. 
simultaneously over both the regions. This suggests that two distinct mechanisms may bring about cartilage destruction in this compartment. Such details are missing from publications on double contrast arthrography by Thomas et al, ${ }^{13}$ Butt et al, ${ }^{23}$ and Staple, ${ }^{24}$ presumably because of the lack of magnification when using conventional radiography.

The present study further stresses the value of weight bearing views when assessing cartilage changes in OA knees. In addition, it reveals a new finding: namely, that the JSW in the middle region of both compartments is smaller than the sum of the cartilage thicknesses. The radioanatomical meaning of this finding becomes apparent when we take into account the fact that the films used to measure JSW were obtained in the weight bearing position, while those used to measure the cartilage thicknesses were obtained in the non-weight bearing position. In the weight bearing tunnel view, the femoral condyle may be expected to exert the greatest load across the middle region of the tibial plateau, ${ }^{19}$ therefore the articular cartilage over the middle region in both the compartments can be expected to be compressed. Thus, depending upon the stage of OA disease in the compartment, and the extent to which the biophysical properties of cartilage may have been altered, JSW will reliably reflect cartilage compression and thinning.

Utilising high definition $x$ ray equipment we have shown that standardised joint position, radiography and mensural procedures help to measure cartilage thickness accurately and reliably as JSW. Similar attention to detail would potentially improve the accuracy and reliability of JSW measurements using conventional radiography.

This work was supported by a grant from Ciba Geigy Pharmaceuticals, UK. The authors wish to express their gratitude to Mrs Alison Robins for her assistance during the radiographic procedures, to Mrs Sheila Bishop for typing the manuscript and Mr Kevin Fitzpatrick and Miss Sarah Smith for manuscript and Mr Kevin Fitzpat
their photographic assistance.

1 Altman R, Fries J F, Block D A, et al. Radiologic assessment of progression in osteoarthritis. Arthritis Rheum 1987; 30: of progressio.

2 Fife R S, Brandt K D, Braunstein E M, et al. Relationship between arthroscopic evidence of cartilage damage and radiographic evidence of joint space narrowing in early osteoarthritis of the knee. Arthritis Rheum 1991; 34 377-82.

3 Brandt K D, Fife R S, Braunstein E M, Katz B. Radiographic grading of knee osteoarthritis: relation of the Kellgren and Lawrence grade to a grade based on joint space narrowing, and correlation with arthroscopic evidence of articular cartilage degeneration. Arthritis Rheum 1991; 34: 1381-6.

4 Buckland-Wright J C, Macfarlane D G, Jasani M K, Lynch J A. Quantitative microfocal radiographic assessment of osteoarthritis of the knee from weight bearing tunnel and semi-flexed standing views. $\mathcal{f}$ Rheumatol 1994; 21: 1734-41.

5 Buckland-Wright J C. A new high-definition microfocal X-ray unit. Br F Radiol 1989; 62: 201-8.

6 Buckland-Wright J C, Bradshaw C R. Clinical applications of high-definition microfocal radiography. $\mathrm{Br} \mathcal{F}$ Radiol 1989; 62: 209-17.

7 Jonsson K, Buckwalter K, Helvie M, Niklason L, Martel W. Precision of hyaline cartilage thickness measurements. Acta Radiol 1992; 33: 234-9.

8 Buckland-Wright J C. Quantitative radiography of osteoarthritis. Ann Rheum Dis 1994; 53: 268-75.

9 Kellgren J H, Lawrence J S. Radiological assessment of osteoarthrosis. Ann Rheum Dis 1957; 16: 494-501.

10 Dacre J E, Scott D L, Da Silva J A P, Welsh G, Huskisson $\mathrm{E} C$. Joint space in radiologically normal knees. $B r f$ Rheumatol 1991; 30: 426-8.

11 Karvonen R I, Negendank W G, Teitge R A, Reed A H, Miller P R, Fernandez-Madrid F. Factors affecting articular cartilage thickness in osteoarthritis and ageing. f Rheumatol 1994; 21: 1310-8.

12 Lynch J A, Buckland-Wright J C, Macfarlane D G. Precision of joint space width measurement in knee osteoarthritis from digital image analysis of high definition macroradiographs. Osteoarthritis Cartilage 1993; 1: 209-18.

13 Thomas R H, Resnick D, Alazraki N P, Daniel D, Greenfield R. Compartmental evaluation of osteoarthritis of the knee. A comparative study of available diagnostic modalities. Radiology 1975; 116: 585-94.

14 Sartoris D J, Resnick D. Plain film radiography: routine and specialized techniques and projections. In: Resnick D, specialized techniques and projections. In: Resnick D, Niwayama G, eds. Diagnosis of bone and joint disord

15 Spring M W, Buckland-Wright J C. Contrast medium imbibition in osteoarthritic cartilage. Brf Radiol 1990; 63: 823-5.

16 Buckland-Wright J C, Carmichael I, Walker S R. Quantitative microfocal radiography accurately detects oint changes in rheumatoid arthritis. Ann Rheum Dis 1986; 45: 463-7.

17 Norusis M J. SPSS/PC+ for the IBMPC/XT/AT. Chicago: SPSS Inc., 1986

18 Campbell M J, Machin D. Medical statistics: a commonsense approach. New York: John Wiley, 1990; 82-6.

19 Simkin P A, Gravey D O, Fiechtner J J. Roman arches, human joints and disease. Arthritis Rheum 1980; 23: 1308-11.

20 Unsworth A. Biomechanics of articulations. Brf Rheumatol 1989; 28: 446-50.

21 Bullough P G, Walker P S. The distribution of load through the knee joint and its possible significance to the observed patterns of articular cartilage breakdown. Bull Hosp foint Dis Orthop Instit 1977; 37: 110-23.

22 Kelly M A, Fithian D C, Chern K Y, Mow V C. Structure and function of the meniscus: basic and clinical implications. In: Mow V C, Ratcliffe A, Woo S L Y, eds. Biomechanics of diarthrodial joints, Vol. 1. New York: Springer-Verlag, 1990: 90-211.

23 Butt W P, McIntyre J L. Double contrast arthrography in the knee. Radiology 1969; 92: 487-99.

24 Staple T W. Extrameniscal lesions demonstrated by double contrast arthrography of the knee. Radiology 1972; 102: $311-9$. 